\title{
Interactive comment on "Evaluation of Arctic sea-ice drift and its dependency on near-surface wind and sea-ice concentration and thickness in the coupled regional climate model HIRHAM-NAOSIM" by Xiaoyong Yu et al.
}

Xiaoyong Yu et al.

xiaoyong.yu@awi.de

Received and published: 10 December 2019

The comment was uploaded in the form of a supplement:

https://www.the-cryosphere-discuss.net/tc-2019-183/tc-2019-183-AC1-

supplement.pdf 